\title{
Enlisting potential cathode materials for rechargeable Ca-batteries
}

\author{
Arturo Torres, José Luis Casals, and M. Elena Arroyo-de Dompablo*
}

\section{Supplementary Information}

\section{$\mathrm{Ca}_{3} \mathrm{MnN}_{3}$ ICSD-67888: Voltage $=1.1 \mathrm{~V}$ Capacity $=124 \mathrm{mAh} / \mathrm{g} \quad \Delta$ Volume $=1 \%$}

The structure of $\mathrm{Ca}_{3} \mathrm{MnN}_{3}$ can be described as alternating layers of triangular planes of $\mathrm{Mn}$ ions along de $\mathrm{C}$ axis. In this structure there are two types of $\mathrm{Ca}$ (see Figure S1): $\mathrm{Ca}$ (in orange) and $\mathrm{Ca} 2$ (in cyan). In both cases, the Ca polyhedra are linked by corner sharing. In Cal type, calcium is linked to 5 nitrogen atoms resulting a square basis pyramid with Ca- $N$ distances of: $2.391 \AA, 2.514(\times 2) \AA, 2.590(x 2) \AA$. In Ca2 type, the pyramid of calcium described before has lost a nitrogen atom, so it forms a tetrahedra with the following bonding distances: $2.417 \AA, 2.514(\times 2) \AA$ and $2.490 \AA$.

As there are two different types of calcium ions, three different pathways are possible. Firstly, the Cal-Ca1 pathway, in the cb plane, where the distance $\mathrm{Ca}-\mathrm{Ca}$ is $3.132 \AA$. The calculated energy barrier is $2.2 \mathrm{eV}$ (Figure S1). A second pathway, between $\mathrm{Ca} 2$ ions at a distance of $3.271 \AA$, has an associated a barrier energy of $1.2 \mathrm{eV}$. In the third pathway, diffusion from Ca1 to Ca2 (ab plane) also has a large energy barrier of $2.1 \mathrm{eV}$.

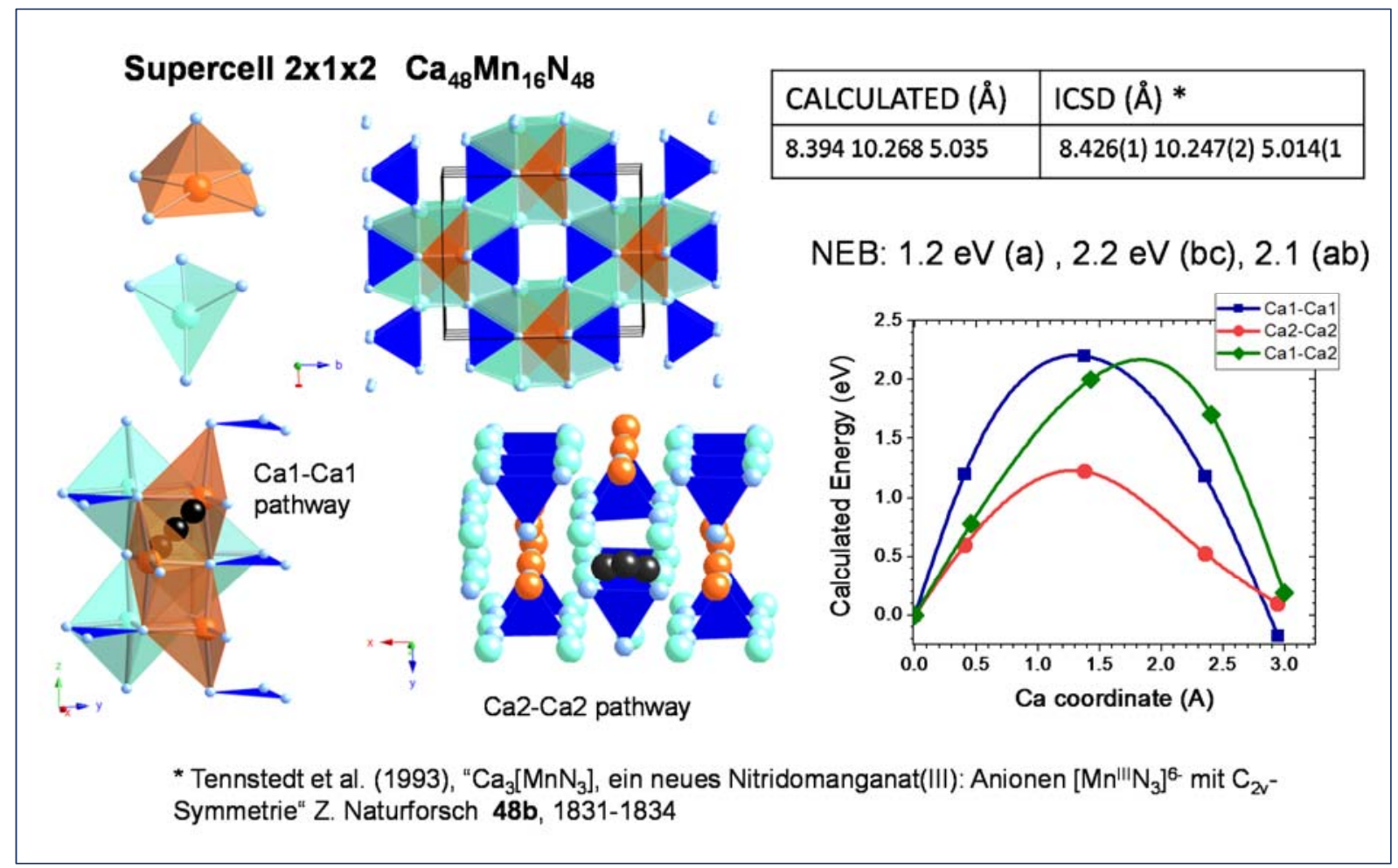

Figure S1. Summary of NEB calculations for $\mathrm{Ca}_{3} \mathrm{MnN}_{3}$. Color code: Ca1 orange, Ca2 cyan, Mn blue, N light grey and diffusing Ca black. 


\section{CaNiN ICSD-69044: Voltage $=2.1 \mathrm{~V}$ Capacity $=475 \mathrm{mAh} / \mathrm{g} \quad \Delta$ Volume $=17 \%$}

The uncommon structure of CaNiN (S.G. P4 $/ \mathrm{mmc}$ ), is characterized by chains of linearly coordinated $\mathrm{Ni}$ ions - $\mathrm{N}-\mathrm{Ni}-\mathrm{N}-\mathrm{Ni}$-. The chains are stacked in the way that there are layers of parallel chains, but chains in adjacent layers run in perpendicular directions. Calcium occupies the tetrahedral sites between the layers, with Ca-N distances of $2.596 \mathrm{~A}$. Interestingly, the nitrogen atoms are in approximately octahedral coordination, with two nickel and four calcium near-neighbour atoms.

Ca migration has been investigated in a $3 \times 3 \times 2$ supercell of CaNiN. We have studied two different cases of diffusion (Figure S2). The first one is set on the a axis, between two calcium atoms separated by $3.356 \AA$ (is the same by geometry on b axis). In this pathway, the Ca ion jumps between the equilibrium sites crossing triangular faces to the intermediate tetrahedral site. The calculated energy barrier for migration barrier is $1.2 \mathrm{eV}$. The second diffsuion pathway is in the c axis, where the distance between both calcium atoms is $3.31 \mathrm{~A}$. Calcium must diffuse by the $\mathrm{N}-\mathrm{N}$ edge, hence causing a higher energy value (1.9 eV).

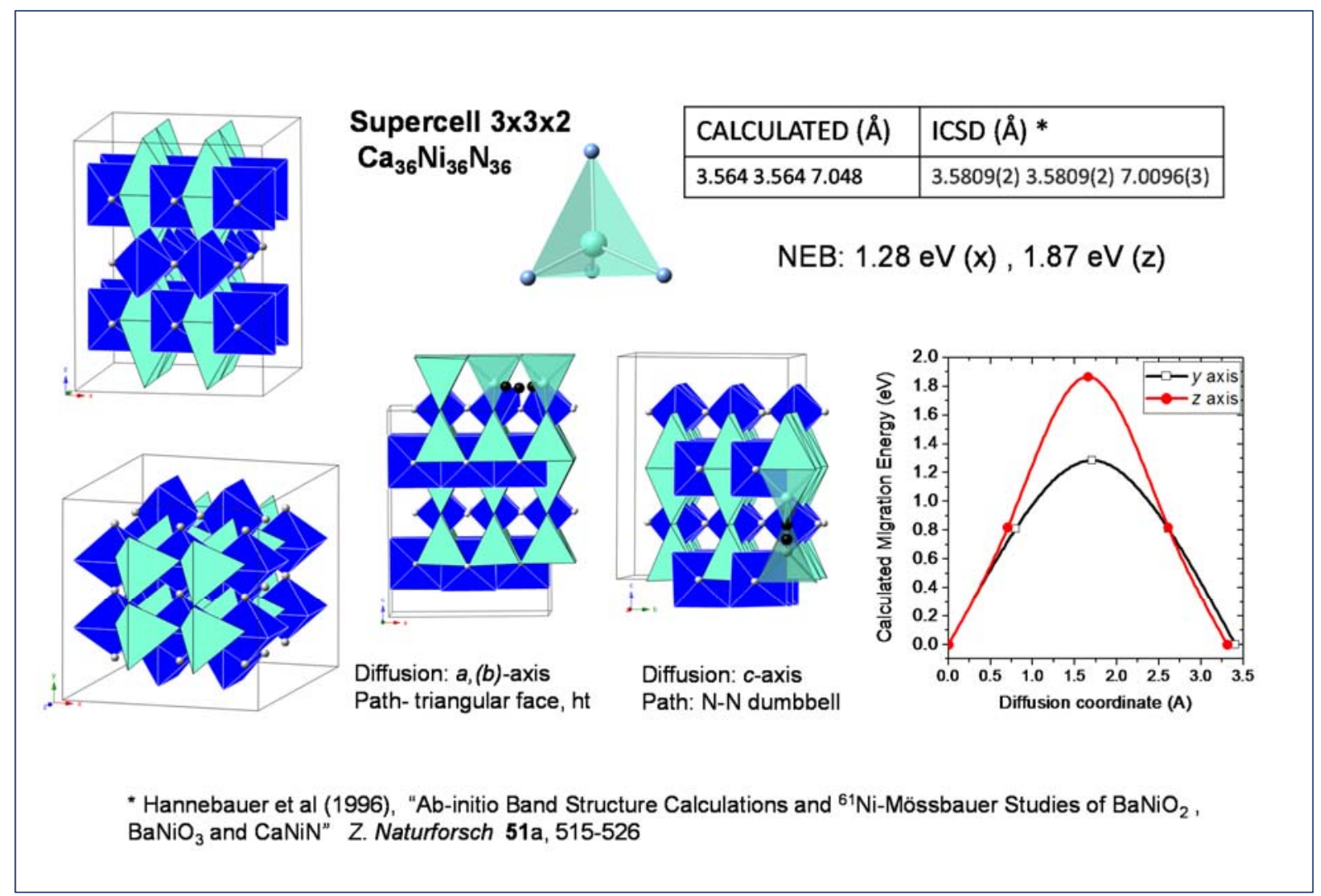

Figure S2. Summary of NEB calculations for CaNiN. Code: Ca cyan, Ni blue, N white, diffusing Ca black. 

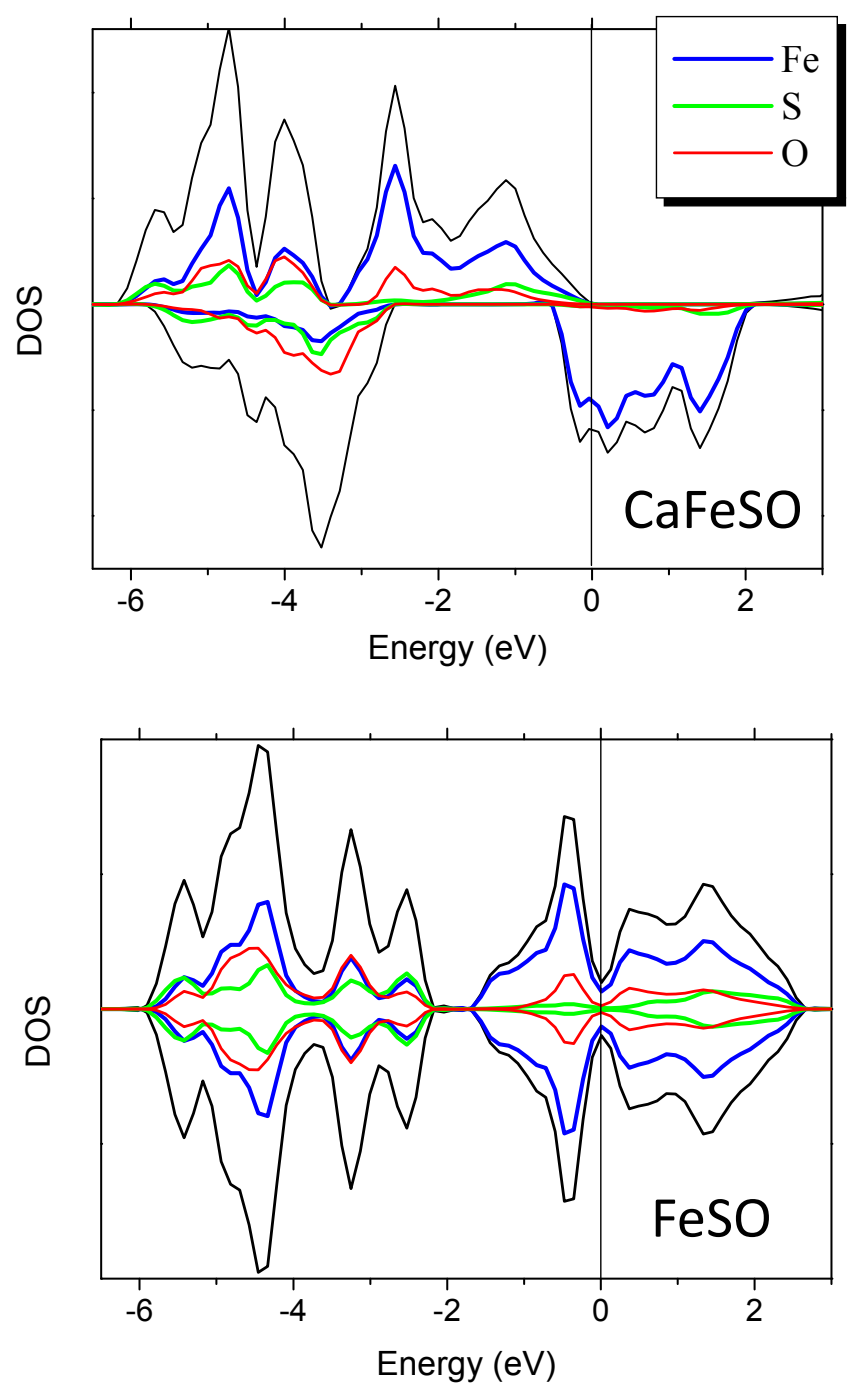

Figure S3. Calculated Density of States of CaFeSO and FeSO (GGA data). The Fermi level is set as the zero of energy. The $\mathrm{Fe}^{2+}$ ions in CaFeSO ( $d^{6}$ configuration) oxidize to diamagnetic $\mathrm{LS}^{-\mathrm{Fe}^{4+}}\left(\mathrm{d}^{4}\right.$ configuration). Within the GGA+U approximation $\mathrm{HS}-\mathrm{Fe}^{4+}$ is predicted in FeSO. 


\section{$\mathrm{CaMn}\left(\mathrm{P}_{2} \mathrm{O}_{7}\right)$ ICSD-78659: Voltage $=4.6 \mathrm{~V}$ Capacity $=154 \mathrm{mAh} / \mathrm{g} \quad \Delta$ Volume $=\mathbf{5} \%$}

The structure of $\mathrm{CaMn}\left(\mathrm{P}_{2} \mathrm{O}_{7}\right)$ is formed of alternating layers of $\mathrm{P}_{2} \mathrm{O}_{7}$ and $\mathrm{Mn}_{2} \mathrm{O}_{10}$ groups, creating cavities in which the $\mathrm{Ca}^{2+}$ cations are found. The upper panel of Figure S3 shows two views of the crystal structure. The $\mathrm{Mn}^{2+}$ ions are in octahedral coordination, and arranged in pairs through the formation of $\mathrm{Mn}_{2} \mathrm{O}_{10}$ dinuclear groups in which two octahedra share a common edge. $\mathrm{P}_{2} \mathrm{O}_{7}$ groups exist in eclipsed conformation.

Although the calculated average intercalation voltage (above $4 \mathrm{~V}$ ) might be too high for the current electrolytes, we have performed a fast and preliminary investigation of the Ca mobility in this structure. To do so, we have performed a single-image NEB calculations for $M=M n$, using a $2 \times 2 \times 2$ supercell $\left(\mathrm{Ca}_{16} \mathrm{Mn}_{16} \mathrm{P}_{32} \mathrm{O}_{102}\right.$ ). In $\mathrm{CaMn}_{2} \mathrm{P}_{2} \mathrm{O}_{7}$, the $\mathrm{Ca}$ ions display seven-fold coordination with average $\mathrm{Ca}-\mathrm{O}$ distance equal to $2.474 \AA$, and one $\mathrm{O}$ ion at a substantially longer distance of $3.053 \AA$. Along the a axis there Ca rows with $\mathrm{Ca}-\mathrm{Ca}$ distances of $6.5 \AA$, but with the intermediate site in between two Ca ions showing short contacts to $\mathrm{Mn}$ and $\mathrm{P}$ ions. In the cb plane, the distances between $\mathrm{Ca}$ ions belonging to different rows are of $3.6 \AA$ and $5.6 \AA$. The calculated energy barriers for the two pathways considered in the cb plane (see figure S4) are $1.2 \mathrm{eV}$ and $2.2 \mathrm{eV}$.

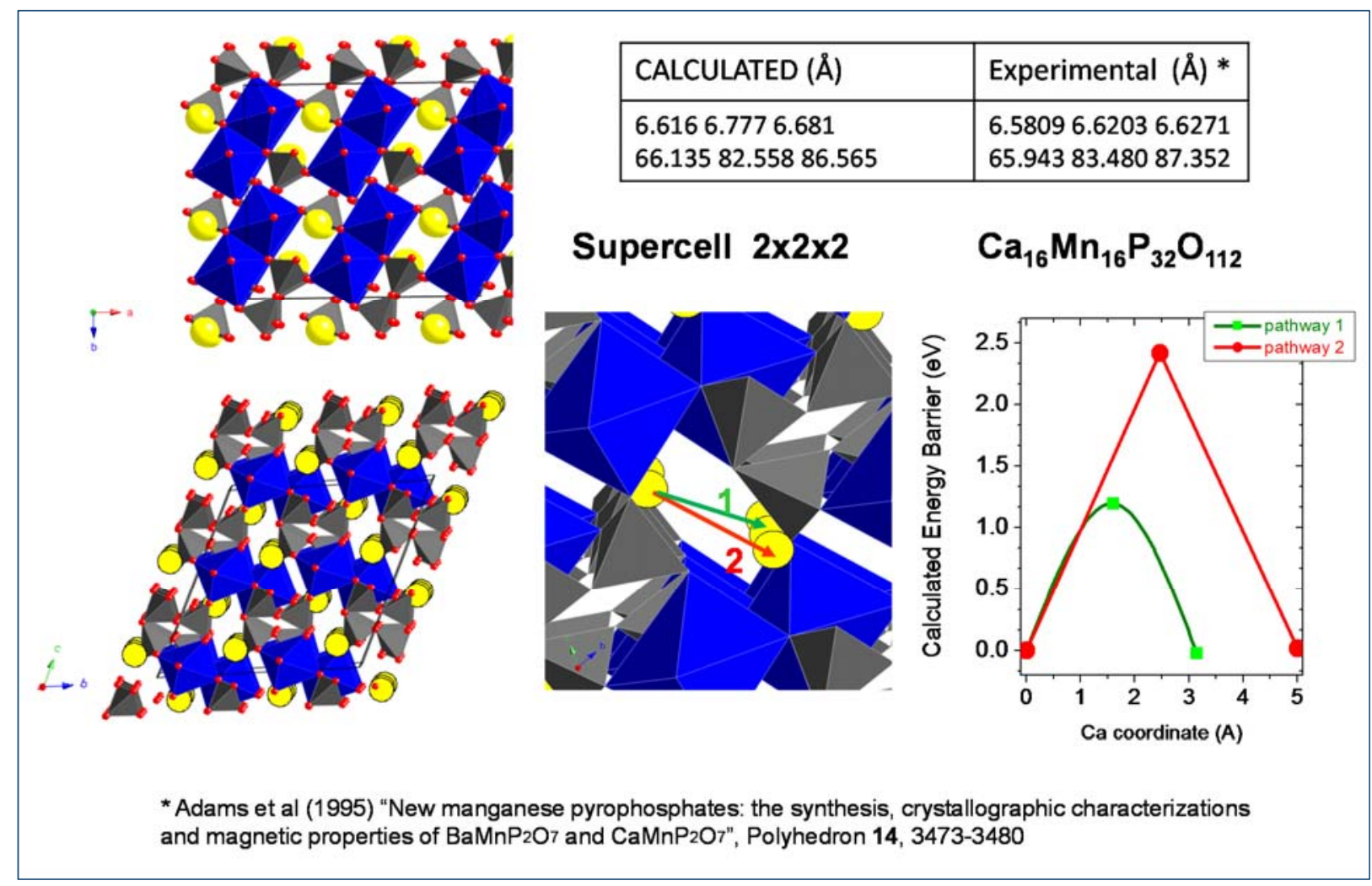

Figure S4. Summary of $\mathrm{NEB}$ calculations for $\mathrm{CaMnP}_{2} \mathrm{O}_{7}$. Color code: Ca yellow, $\mathrm{V}$ blue, Si grey, O red, intermediate $\mathrm{Ca}$ site in the diffusion pathway in green. 


\section{Evaluation of $\mathrm{CaV}_{2} \mathrm{O}\left(\mathrm{PO}_{4}\right)_{2}$ ICSD-79735}

The structure of the $\mathrm{V}^{3+}$ monophosphate $\mathrm{CaV}_{2} \mathrm{O}\left(\mathrm{PO}_{4}\right)_{2}$ consists of $\left[\mathrm{V}_{2} \mathrm{PO}_{7}\right.$ ] layers, built up from [ $\mathrm{VO}_{6}$ ] rutile chains and $\left[\mathrm{VPO}_{8}\right]$ chains, interconnected through $\mathrm{PO}_{4}$ tetrahedra (Figure S5). This framework delimits tunnels running along $(011]$, at the intersection of which the calcium ions are located. This cation, exhibits distorted octahedral coordination, with Ca-O distances ranging from 2.342 to $2.519 \AA$, but with a seventh neighbor at $2.911 \AA$. As in $\mathrm{Ca}(\mathrm{VO})_{2}\left(\mathrm{PO}_{4}\right)_{2}$ the $\mathrm{Ca}$ atoms are far apart (Ca-Ca distance is 4.94 $\AA$ ). Figure S5 shows the possible pathway for $\mathrm{Ca}$ diffusion. The intermediate $6+1$ coordinated site shares a face with the $\mathrm{VO}_{6}$ octahedra and shares an edge with another $\mathrm{VO}_{6}$ octahedra and two $\mathrm{PO}_{4}$ tetrahedra. An additional penalty for Ca mobility is the triangular window in the Ca equilibrium site (note that in $\mathrm{Ca}(\mathrm{VO})_{2}\left(\mathrm{PO}_{4}\right)_{2}$ the window is quadrangular). In view of previous results this pathway seems unlikely for fast Ca diffusion.
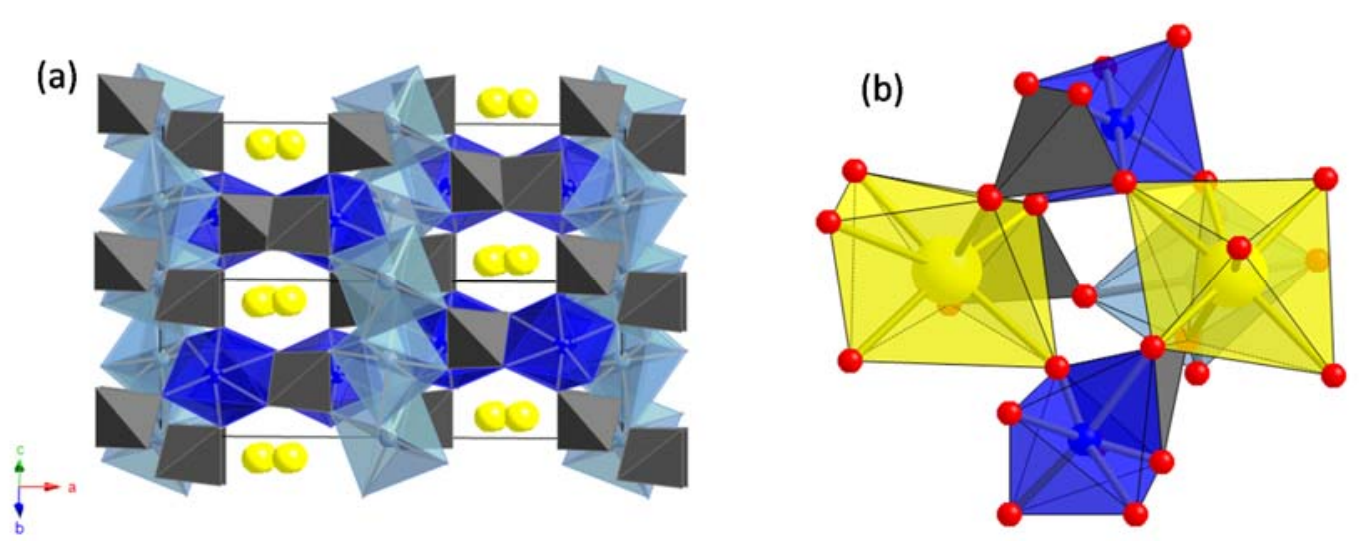

Figure S5. The crystal structure of $\mathrm{CaV}_{2} \mathrm{O}\left(\mathrm{PO}_{4}\right)_{2}$. (a) three-dimensional $\mathrm{V}-\mathrm{P}-\mathrm{O}$ framework (c) Possible pathway for $\mathrm{Ca}$ diffusion. Note the triangular windows in the " $6+1$ " coordinated ca ions. Color code Ca in yellow, $\mathrm{V}$ in blue, $\mathrm{V}$ in rutile chains light blue, $\mathrm{P}$ in grey and $\mathrm{O}$ in red.

\section{$\mathrm{Ca}(\mathrm{VO})_{2}\left(\mathrm{PO}_{4}\right)_{2}$ ICSD-728864: Voltage $=4.2 \mathrm{~V}$ Capacity $=147 \mathrm{mAh} / \mathrm{g} \Delta \mathrm{Vol} .=3 \%$}

The structure of $\mathrm{Ca}(\mathrm{VO})_{2}\left(\mathrm{PO}_{4}\right)_{2}$ consists of infinite chains of trans-corner-sharing $\mathrm{VO}_{6}$ octahedra crosslinked by phosphate groups to form a 3-dimensional network (Figure S5a). These chains have alternating short and long VO bonds (Figure S6b). The V-P-O network contains intersecting channels to [100] and [001] where the $\mathrm{Ca}^{2+}$ cations are located. The $\mathrm{Ca}$ ions are 8-coordinated to oxygen (average Ca-O bond length at $2.528 \AA$ ) and share edges and corners with the $\mathrm{PO}_{4}$ and $\mathrm{VO}_{6}$ polyhedra. The $\mathrm{Ca}$ ions are far apart (distance $\mathrm{Ca}-\mathrm{Ca}$ is $5.24 \AA$ ), and possible $\mathrm{Ca}$ diffusion may occur across the intermediate eight-fold coordinated site that is empty (Figure S6c). The calculated energy barrier for Ca migration is 3 eV (Figure S7), as a result of the short Ca-P distances (2.56-2.71 $\AA$ ) at the intermediate site. Hence, regardless the theoretical energy of $597 \mathrm{mWh} / \mathrm{g}$ (calculated voltage $4.2 \mathrm{~V}$, specific capacity for $\mathrm{V}^{4+} / \mathrm{V}^{5+}=142 \mathrm{mAh} / \mathrm{g}$ ) the material was discarded for experimental investigation. 

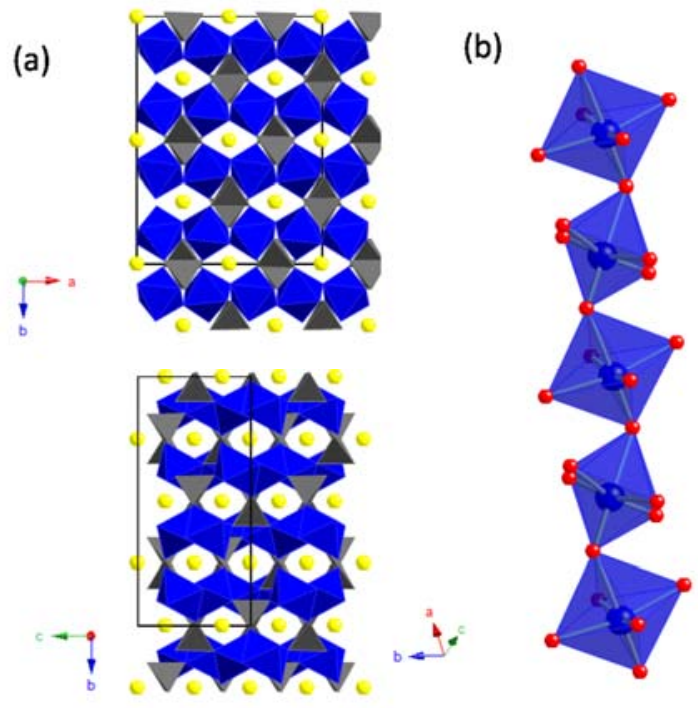

(c)

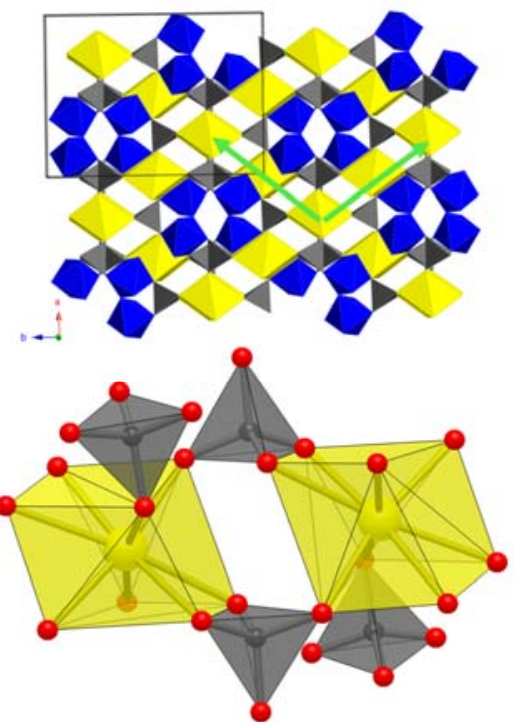

Figure S6. The crystal structure of $\mathrm{Ca}(\mathrm{VO})_{2}\left(\mathrm{PO}_{4}\right)_{2}$. (a) three dimensional V-P-O framework (b) $\mathrm{VO}_{6}$-chains and (c) pathways for $\mathrm{Ca}$ diffusion. Color code $\mathrm{Ca}$ in yellow, $\mathrm{V}$ in blue, $\mathrm{P}$ in grey and $\mathrm{O}$ in red.

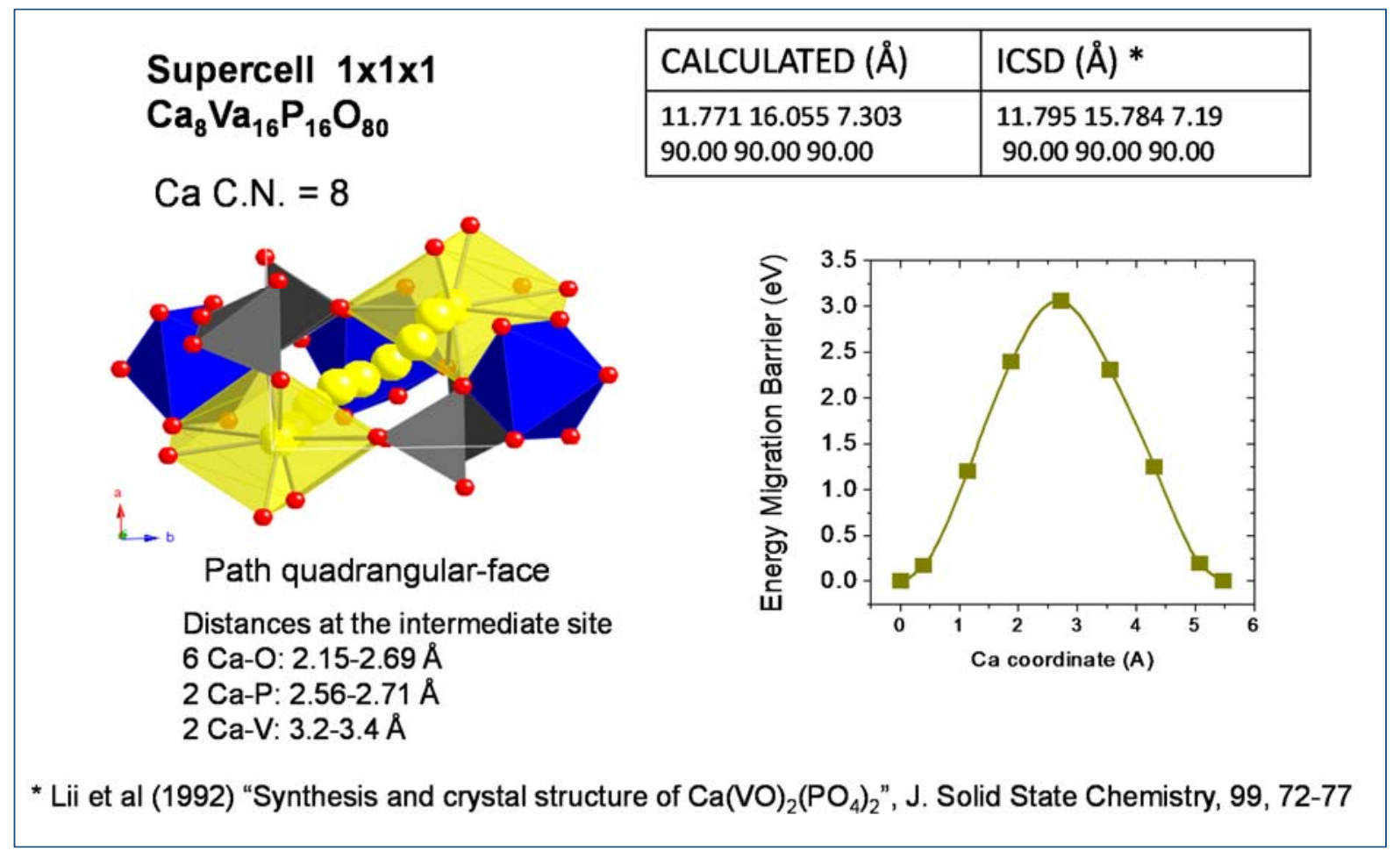

Figure S7. Summary of NEB calculations for $\mathrm{Ca}(\mathrm{VO})_{2}\left(\mathrm{PO}_{4}\right)_{2}$ Color code: Ca yellow, V blue, Si grey, O red, diffusing Ca yellow balls. 